\title{
Statistical microdamage mechanics and damage field evolution
}

\author{
Y.L. Bai ${ }^{\text {a,* }}$, M.F. Xia ${ }^{\text {a,b }}$, F.J. Ke ${ }^{\text {a,c }}$, H.L. Li ${ }^{\text {a }}$ \\ a State Key Laboratory for Nonlinear Mechanics (LNM), Institute of Mechanics, Chinese Academy of Sciences, \\ Beijing 100080, People's Republic of China \\ b Department of Physics, Peking University, Beijing 100871, People's Republic of China \\ ${ }^{\mathrm{c}}$ Department of Applied Physics, Beijing University of Aeronautics and Astronautics, Beijing 100083, People's Republic of China
}

\begin{abstract}
Discussed are the underlying background of statistical microdamage mechanics, the fundamental partial differential equation of evolution of microdamage number density, two basic solutions, and the saturation of microdamage number density evolution. Knowledge of microdamage number density evolution is applied to engineering practice by using the field equations of microdamage number density and continuum damage. The addition of continuum equations renders a complete system of field equations of deformation and damage. However, they are open-ended in character at the continuum level although the dynamic damage function is completed from the meso- to the macro-scale level. Once decoupling of the function is made, the system of equations can be connected in an approximate manner. This provides a reasonable approximation to the continuum field of deformation and damage. The open literature prediction based on damage evolution relies on assuming arbitrary critical damage states. In this work, use is made of the criterion for damage localization. Several applications of statistical microdamage mechanics are made. This includes damage evolution in a heterogeneous medium and failure forecast under impact. The results show that statistical microdamage mechanics and the derived closed approximate continuum formulations are physically sound and practically effective. (c) 2001 Elsevier Science Ltd. All rights reserved.
\end{abstract}

\section{Background of statistical microdamage mechan- ics}

When a large crack dominates in a solid, fracture mechanics (linear elastic or elastic-plastic) can be used to characterize the mechanical behavior of solids. However, for most heterogeneous materials, such as alloys, ceramics, composites, rocks, etc., there might be distributed microcracks or microvoids rather than a single macroscopical crack. In particular, it is well known that distributed microdamages usually dominate failure of

\footnotetext{
${ }^{*}$ Corresponding author.

E-mail address: baiyl@lnm.imech.ac.cn (Y.L. Bai).
}

solids under various loadings such as impact. Instead of fracture mechanics, continuum damage mechanics may be more appropriate [1-5]. The essence of continuum damage mechanics consists of evolution and a critical damage parameter $D_{\mathrm{c}}$. An evolution law is usually assumed in the form

$$
\dot{D}=\frac{K}{(1-D)^{\gamma}},
$$

where $\gamma$ is an undetermined parameter and $K$ is a stress-dependent function $K(\sigma)$. The nominal stress $\sigma$ in the damaged solid is assumed to be related to the stress $\sigma_{\mathrm{s}}$ in the intact portion of the materials:

$$
\sigma=\sigma_{\mathrm{s}}(1-D) \text {. }
$$


To forecast failure, a critical damage state should be assumed. Suggested in [4] is the range of $D_{\text {c }} \sim 0.2-0.5$.

As a matter of fact, the continuum damage $D$ should be a collective representation of microdamage [5-10], Fig. 1. The evolution of damage should be based on physics and a critical damage $D_{\text {c }}$ which is not arbitrarily assumed. It can be quoted from ASTM [8] that "rigorous treatment of non-uniformly distributed defects requires tools not yet fully developed in continuum damage mechanics. Weighing the influence of distributed damage at the microscale on the collective macroscale stiffness and evolution of damage is a challenge as well. In fact, the overall framework of continuum damage mechanics, based on the use of internal state variables to represent evolving structure of the material, appeals strongly to irreversible statistical thermodynamics.

Generally speaking, microdamage gives rise to inhomogeneities such as particulates or grains of the order of micrometres. On the metal surface, the microdamage is in the range $10^{2}-10^{4} / \mathrm{mm}^{2}$ $[6,10]$. Some of the microdamages may grow and coalesce causing eventual failure. Hence, the main issues in microdamage evolution are nucleation, growth and coalescence of microdamage [6-10]. Hence, there is a need to understand trans-scale (from meso- to macroscopic) damage evolution.

This work focuses on the basic concepts and fundamentals of statistical microdamage mechanics. Included are the equations of microdamage evolution and their solutions. Because of the separate division of meso- and macroscopic entities, effort is made to reduce the discrete nature of the governing equations. A criterion for damage localization is used to forecast failure. Statistical

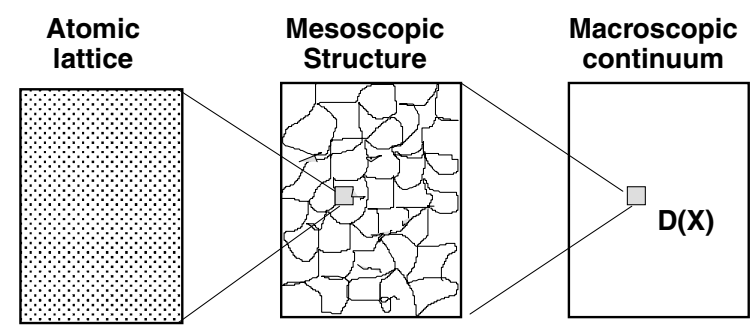

Fig. 1. Schematic of length scales. microdamage mechanics is applied to a prototyped heterogeneous medium and failure under impact loading. It is shown that statistical microdamage mechanics and the corresponding approximate formulation at the continuum level yield realistic results.

\section{Evolution of microdamage number density}

\subsection{Fundamental equation}

A framework of statistical microdamage mechanics [10] is established. An evolution equation of microdamage number density $n$ given by

$\frac{\partial n}{\partial t}+\sum_{i=1}^{l} \frac{\partial\left(n \cdot P_{i}\right)}{\partial p_{i}}=n_{\mathrm{N}}-n_{\mathrm{A}}$

is introduced in the phase space $\left\{p_{i}\right\}$ (see Fig. 2), where $t$ is the generalized time. $P_{i}=\dot{p}_{i}$ are the rates of variables $p_{i} . n_{\mathrm{N}}$ and $n_{\mathrm{A}}$ are nucleation and annihilation rate densities of microdamage, respectively. For simplicity, ignore the term of annihilation.

Phase space of microdamage consists of variables $p_{i}$, such as the size and orientation of microdamage and the macroscopic position where microdamage is located, etc. Here, confinement is made to the phase space $\left\{c, c_{0}, \boldsymbol{x}\right\}$ of current size $c$ and initial size $c_{0}$ of microdamage and its macroscopic position $\boldsymbol{x}$. The product of number density of microdamage $n(c, \boldsymbol{x})$ and element volume in

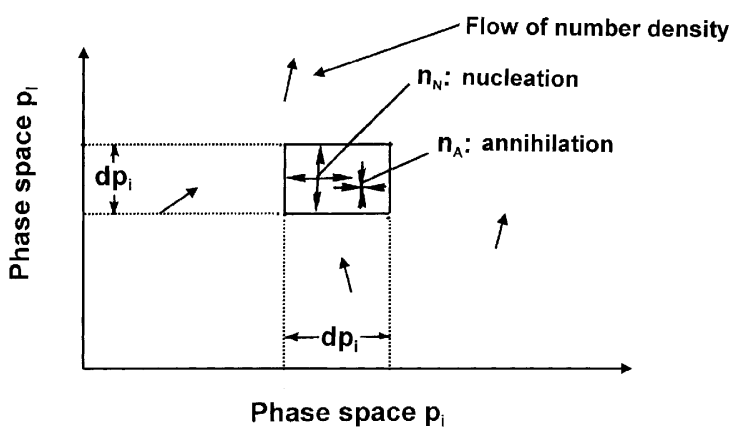

Fig. 2. Schematic of phase space of microdamage; derivation of the fundamental evolution equation of microdamage number density $n$. 
phase space $\mathrm{d} c \cdot \mathrm{d} \boldsymbol{x}$ corresponds to the total microdamage with the current size from $c$ to $c+\mathrm{d} c$ at the macroscopic position from $\boldsymbol{x}$ to $\boldsymbol{x}+\mathrm{d} \boldsymbol{x}$. For instance, $n(c, \boldsymbol{x})=10 / \mu \mathrm{m} \mathrm{mm^{3 }}$ means that a volume of $1 \mathrm{~mm}^{3}$ at macroscopic point $\boldsymbol{x}$ has altogether 10 microdamages with size from $c$ to $c+1 \mu \mathrm{m}$.

\subsection{Basic solutions}

Two basic solutions $n(t, c ; \sigma)$ and $n_{0}\left(t, c, c_{0} ; \sigma\right)$ are obtained from the fundamental equation of microdamage number density (3) in phase spaces $\{c\}$ and $\left\{c, c_{0}\right\}$ with constant stress parameter $\sigma$. The details can be found in [11-13]. The first solution [11] is given by

$n(t, c ; \sigma)= \begin{cases}n_{\mathrm{N}}(c ; \sigma) \cdot t, & c<b, \\ \int_{\eta(t, c)}^{c} n_{\mathrm{N}}\left(c^{\prime} ; \sigma\right) \cdot \mathrm{d} c^{\prime} / A(c ; \sigma), & c \geqslant b\end{cases}$

where the growth rate

$\dot{c}=\left\{\begin{array}{ll}0, & c<b, \\ A(c ; \sigma), & c \geqslant b,\end{array}\right.$ and $t=\int_{\eta(t, c)}^{c} \frac{\mathrm{d} c^{\prime}}{A\left(c^{\prime} ; \sigma\right)}$.

The distinct aspect of this solution is that for a given microdamage size $c>b$, when $t>t_{0}=\int_{b}^{c} \mathrm{~d} c^{\prime} / A\left(c^{\prime} ; \sigma\right)$, microdamage number density will become saturated. In other words, for a given time $t$ there is a saturation front $c_{\mathrm{s}}$ of microdamage, defined by $t=\int_{b}^{c_{\mathrm{s}}} \mathrm{d} c^{\prime} / A\left(c^{\prime} ; \sigma\right)$, Fig. 3 . In this situation, the contribution of the microdamage less than $c_{\mathrm{s}}$ and greater than $b$ remains unchanged. Damage evolution will be governed mainly by microdamage greater than $c_{\mathrm{s}}$. This phenomenon is attributed to the incoming and outgoing flux of microdamage across the element as governed by the balance of nucleation in the element $\mathrm{d} c$ in phase space $\{c\}$. The second solution [13] is

$n_{0}\left(t, c, c_{0} ; \sigma\right)=\frac{n_{\mathrm{N}}\left(c_{0} ; \sigma\right)}{V\left(c, c_{0} ; \sigma\right)}$

when $c<c_{\mathrm{f}}$ and $n_{0}=0$

when $c>c_{\mathrm{f}}$,

with a moving front $c_{\mathrm{f}}$. Note that $t=\int_{c_{0}}^{c_{\mathrm{f}}} \mathrm{d} c^{\prime} /$ $V\left(c^{\prime}, c_{0} ; \sigma\right)$, where $V$ is the growth rate of micro-

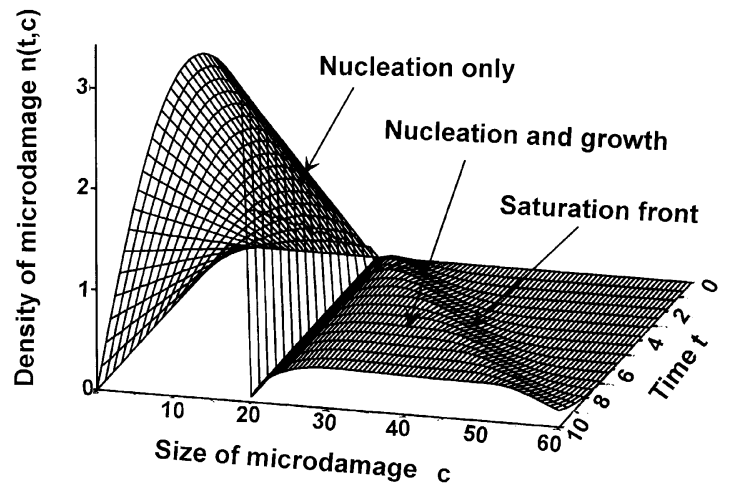

Fig. 3. Solution of number density of microdamage $n(t, c)$ under constant stress. All variables are dimensionless and numbers shown on axes are divisions of dimensionless maximum. Notice that high number density of microdamage within the region where growth rate is zero (left) and the front of saturation of microdamage number density moving rightward.

damage. In most situations, the growth rate of microdamage under a given stress $\sigma$ is not only a function of microdamage size $c$ only but also depends on a binary function of the current size $c$ and original size $c_{0}$ of microdamage, $V\left(c, c_{0} ; \sigma\right)$. Another interesting aspect of this solution is that eventhough the right-hand side of Eq. (5) appears to be in a steady state, it has a time-dependent front $c_{j}$, Fig. 4. Hence, the evolution aspect of microdamage would be contributed mainly made by the moving front $c_{\mathrm{f}}$ only [13]. This reflects the essence of microdamage saturation discussed earlier. The connection between the two solutions becomes clear:

$$
\begin{aligned}
n(t, c ; \sigma) & =\int_{0}^{c} n_{0}\left(t, c, c_{0} ; \sigma\right) \cdot \mathrm{d} c_{0} \\
& = \begin{cases}\int_{0}^{c}\left(n_{\mathrm{N}} / V\right) \cdot \mathrm{d} c_{0}, & c<c_{\mathrm{f}} \\
\int_{0}^{c_{\mathrm{f}}(t)}\left(n_{\mathrm{N}} / V\right) \cdot \mathrm{d} c_{0}, & c>c_{\mathrm{f}}\end{cases}
\end{aligned}
$$

\subsection{Field equations of microdamage number density}

Microdamage evolution will now be examined in terms of inhomogeneous damage field and its effect on eventual failure. As shown in Fig. 1, the number density of microdamage should be dependent on their macroscopic position $\boldsymbol{x}$ as well, 


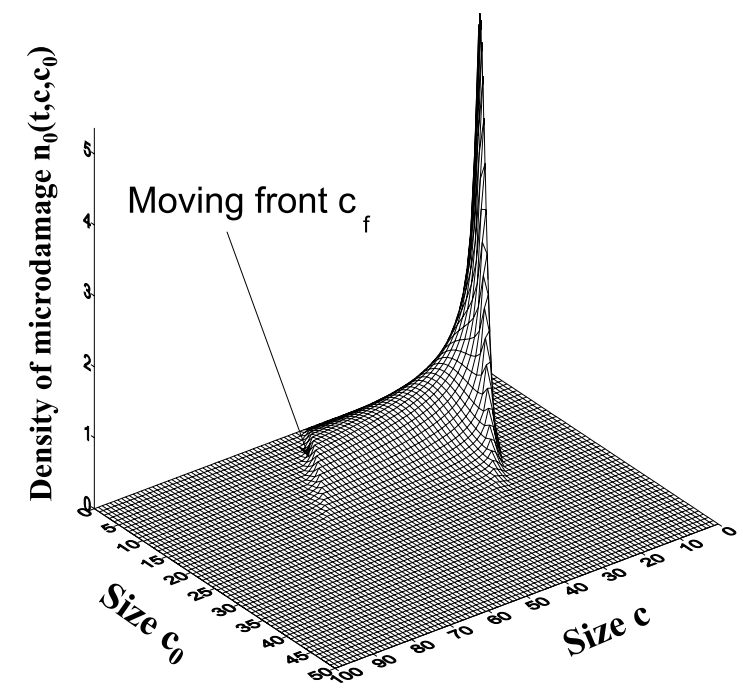

Fig. 4. Solution of number density of microdamage $n_{0}\left(t, c, c_{0}\right)$ under constant stress. All variables are dimensionless and numbers shown on axes are divisions of dimensionless maximum. Notice that the front of microdamage number density moving towards larger size of microdamage $c$ and the steady high number density of microdamage after the front.

namely $n=n(t, \mathbf{x}, c)$. The number density of microdamage is governed by the field equation [14]:

$\frac{\partial n}{\partial t}+\frac{\partial(n \cdot A)}{\partial c}+\frac{\partial(n \cdot \mathbf{v})}{\partial \mathbf{x}}=n_{\mathrm{N}}$.

Clearly, this equation should be related to other continuum equations, such as mass, momentum and energy equations. The coupled nature of this system of equations at the meso- and macroscale level makes the problem of damage field evolution very complicated. For engineering practice, it suffices to connect these equations approximately at the macroscopic level.

\section{Continuum damage field and damage localization}

\subsection{Continuum damage field}

Consider an element of solid that is large enough to contain a number of microdamages as shown in Fig. 1. Make use of the microdamage number density, and define the continuum damage $D$ :
$D(t, \mathbf{x})=\int n(t, \mathbf{x}, c) \cdot \tau \cdot \mathrm{d} c$,

where $\tau$ is the failure volume of an individual microdamage with size $c$. For a spherical microdamage, $\tau \sim \pi c^{3} / 6$. Hence, the field equation of number density of microdamage can be converted to the continuum damage field equation by integration. Application of the appropriate boundary conditions, [14,15] gives

$\frac{\partial D}{\partial t}+\frac{\partial(D \cdot \mathbf{v})}{\partial \mathbf{x}}=f$,

where

$\begin{aligned} f= & \int_{0}^{\infty} n_{\mathrm{N}}(c ; \sigma) \cdot \tau \cdot \mathrm{d} c \\ & +\int_{0}^{\infty} n(t, \mathbf{x}, c) \cdot A(c, \sigma) \cdot \tau^{\prime} \cdot \mathrm{d} c\end{aligned}$

is the dynamic function of damage (DFD). The function bridges the micro- and macrodamage. The entire system of continuum damage and deformation become

$\frac{\partial D}{\partial t}+\mathbf{v} \cdot \frac{\partial D}{\partial \mathbf{x}}+D \cdot \frac{\partial \mathbf{v}}{\partial \mathbf{x}}=f$,

$\frac{\partial \rho}{\partial t}+\mathbf{v} \cdot \frac{\partial \rho}{\partial \mathbf{x}}+\rho \cdot \frac{\partial \mathbf{v}}{\partial \mathbf{x}}=0$,

$\frac{\partial \mathbf{v}}{\partial t}+\mathbf{v} \cdot \frac{\partial \mathbf{v}}{\partial \mathbf{x}}=\rho^{-1} \cdot \frac{\partial \boldsymbol{\sigma}}{\partial \mathbf{x}}$,

$$
\begin{aligned}
& \frac{\partial(e-q)}{\partial t}+\mathbf{v} \cdot \frac{\partial(e-q)}{\partial \mathbf{x}} \\
& \quad=\rho^{-1} \cdot \sigma \cdot \frac{\partial \mathbf{v}}{\partial \mathbf{x}}-\rho^{-1} \cdot \frac{\partial^{2} \mathbf{h}}{\partial \mathbf{x} \cdot \partial \mathbf{x}}
\end{aligned}
$$

where $\rho$ is the density while $e$ and $q$ are the specific internal energy and heat source, respectively. The Cauchy stress tensor is $\boldsymbol{\sigma}$ and $\mathbf{h}$ is the heat flux. The energy equation is dropped for simplicity. For planar impact problem, the one-dimensional strain state will be examined. Hence, all velocity components and spatial derivatives are zero except for the velocity component $\mathrm{v}$ and derivative $(\partial / \partial y)$. The problem is also transformed from the Euclidean $(t, y)$ to the Lagrangian coordinates $(T, Y)$. The transformation is $t=T$ and $y=Y+u$ with $u$ being the displacement or 
$\frac{\partial}{\partial t}+v \cdot \frac{\partial}{\partial y}=\frac{\partial}{\partial T} \quad$ and $\quad \frac{\partial}{\partial y}=\frac{\rho}{\rho_{0}} \cdot \frac{\partial}{\partial Y}$.

Obtained is a system of continuum equations in the one-dimensional strain state:

$\frac{\partial D}{\partial T}+D \frac{\rho}{\rho_{0}} \frac{\partial v}{\partial Y}=f$

$\frac{\partial \rho}{\partial T}+\frac{\rho^{2}}{\rho_{0}} \frac{\partial v}{\partial Y}=0$

$\frac{\partial v}{\partial T}-\rho_{0}^{-1} \frac{\partial \sigma}{\partial Y}=0$

The damage field equation (16) may be regarded as a rate-dependent damage evolution law:

$\dot{D}=\dot{D}(\sigma, \varepsilon, \dot{\varepsilon}, D)=f-D \frac{\dot{\varepsilon}}{1+\varepsilon}$.

Preference is made to Eq. (11) or (16) as a field equation.

\subsection{Closing approximation on continuum level}

The main issue is the meso- and macroscopic coupling in DFD $f$. Note from Eq. (10) that $f$ must depend on stress $\sigma$ and several mesoscopic factors such as nucleation and growth laws, failure volume of microdamage, and number density of microdamage. These quantities are closely related to continuum damage $D$, Eq. (8). An educated guess is that $f$ is dependent on stress $\sigma$ and damage $D$. This is reminiscence of the assumption associated with damage evolution in the internal variable theory of damage mechanics [1-4]:

$\dot{D}=\frac{\partial D}{\partial T}=F(\sigma, D)$.

The problem now is to determine the approximation such that the dependence of function $f$ on nucleation, growth law of microdamage and number density of microdamage would reduce to a single variable $D$. Consider the damage field equation and attempt to find the relation between DFD $f$ and the damage evolution law $F$. Compare the two terms on the right side of Eq. (16) or (19) involving the damage rate and flux. Because of $\frac{\rho}{\rho_{0}}=\frac{1}{1+\varepsilon} \quad$ and $\quad \frac{\partial v}{\partial Y}=\frac{\partial^{2} u}{\partial T \partial Y}=\frac{\partial \varepsilon}{\partial T}$,

an inequality is obtained:

$\frac{\partial D / \partial T}{D} \gg \frac{\partial \varepsilon / \partial T}{1+\varepsilon}$

provided that $D \ll 1$ and $\varepsilon \ll 1$, i.e. small damage and deformation. The damage rate and strain rate are of the same order, i.e., $\dot{\varepsilon} \sim \dot{D}$. These approximations lead to

$\frac{\partial D}{\partial T}=f=F$.

For a fixed macroscopic point $Y$, DFD $f$ can thus be approximated by the damage rate of the point. This is similar to the damage evolution law in the internal variable theory of continuum damage mechanics. The approximate assumption

$f=f(D, \sigma)$.

thus prevails. Here, damage is examined as a field variable, Eq. (16). This is in contrast to the passive dependency of the stress field as in the internal variable theory.

\subsection{Damage localization and Deberah number}

Failure of solids is a highly nonlinear process. A reduction in dimensionality is usually made by considering a two-dimensional fracture surface formed in a three-dimensional body. Of interest is the intrinsic factor that would govern the localized failure. The damage field concept invokes damage localization, Fig. 5:

$\left(\frac{\partial(\partial D / \partial Y)}{\partial T}\right) /\left(\frac{\partial D}{\partial Y}\right) \geqslant\left(\frac{\partial D}{\partial T}\right) / D$.

The interpretation is that the relative rate of damage gradient becomes greater than the relative rate of the damage itself as shown in Fig. 5. For the damage field equation (11) or (16), consider the approximate form $f=f(D, \sigma)$ and differentiate damage field equation (16) with respect to spatial ordinate $Y$. The quasi-static assumption is used to derive the inequality for damage localization [16]; 
(a)

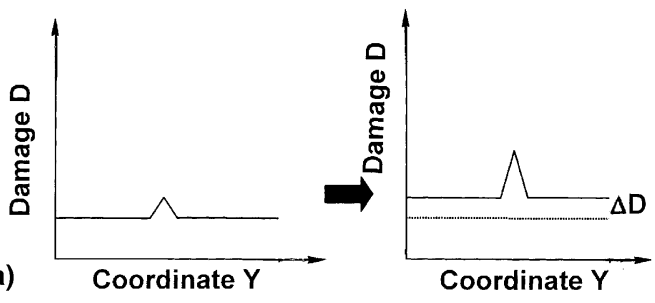

(b)

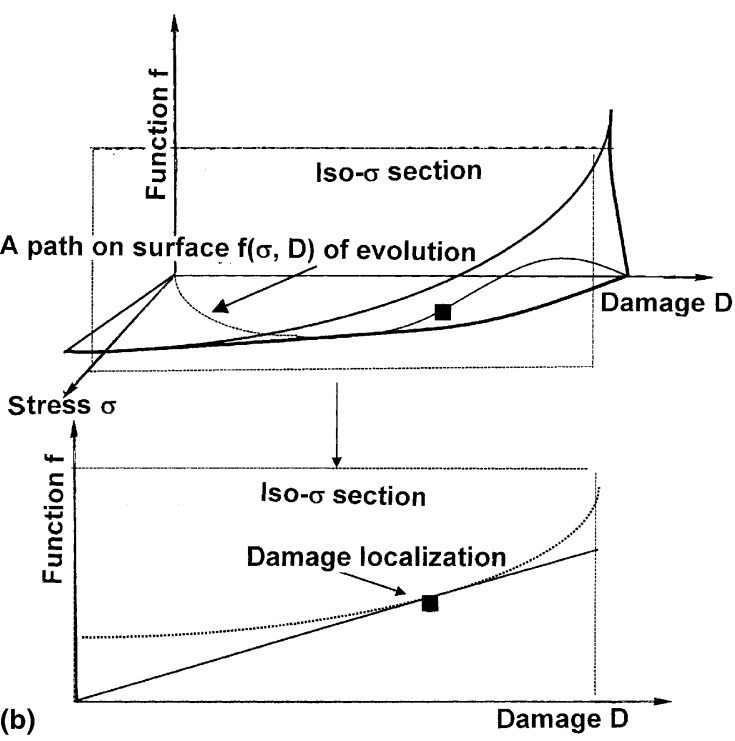

Fig. 5. Damage localization: (a) Geometrical configuration of damage localization. (b) Schematic of the criterion for damage localization $f_{\mathrm{D}}>f / D$.

$f_{\mathrm{D}}-\left(D \frac{\partial\left(\frac{\partial \varepsilon / \partial T}{1+\varepsilon}\right)}{\partial Y}\right) /\left(\frac{\partial D}{\partial Y}\right) \geqslant f / D$.

The second term on the left of Eq. (26),

$$
\left(D \frac{\partial\left(\frac{\partial \varepsilon / \partial T}{1+\varepsilon}\right)}{\partial Y}\right) /\left(\frac{\partial D}{\partial Y}\right)
$$

becomes the order $\mathrm{O}(D)$, provided that

$\frac{\partial(\partial D / \partial Y)}{\partial T} \sim \frac{\partial(\partial \varepsilon / \partial Y)}{\partial T}$

and $\partial D / \partial Y \sim \partial \varepsilon / \partial Y$. Hence, this term can be ignored if damage $D \ll 1$. An approximate criterion for damage localization is thus obtained:

$f_{\mathrm{D}} \geqslant f / D$.
This approximate condition can be easily interpreted and applied in practice according to Fig. 5 when the tangent $f_{\mathrm{D}}$ of DFD on an iso-stress section in coordinates $(f, D, \sigma)$ becomes greater than its secant $f / D$, damage localization will occur. To illustrate the application of the criterion, consider a sample DFD such that

$f=f(D, \sigma)=G(\sigma) \cdot\left(1+\beta \cdot D^{\mu}\right)$,

where $G$ is a function of stress. $\mu$ and $\beta$ are two parameters relevant to damage growth and coalescence. Davison and Stevens [17] have named the two terms in Eq. (28) as simple and compound damages corresponding to nucleation and growth (as well as coalescence) of microdamage, respectively. Generally speaking, the DFD has $\mu>1$ and appears to be concave. In particular, $\beta$ is the ratio of compound rate over nucleation rate of damage. It is an intrinsic Deborah number. In this case, the critical damage for damage localization becomes

$D_{\mathrm{c}}=[(\mu-1) \cdot \beta]^{-1 / \mu}$.

It seems that the index $\mu$ remains in the range 2-3, but Deborah number $\beta$ may change from tens in creep to million in impact. The variation of the critical damage for localization with Deborah number $\beta$ is shown in Fig. 6 .

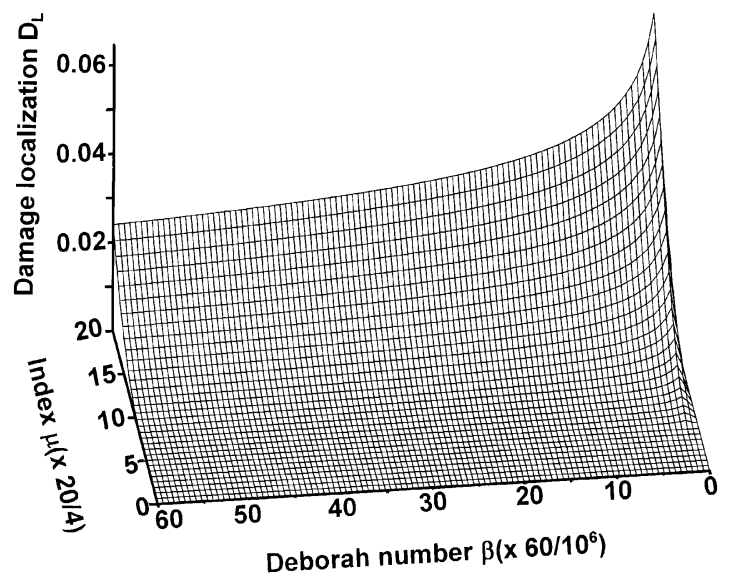

Fig. 6. Critcal damage for damage localization $D_{\mathrm{L}}$ vs index $\mu$ and Deborah number $\beta$. 


\section{Examples}

\subsection{Failure forecast of heterogeneous media}

Mesoscopic heterogeneity is encountered frequently in engineering materials and its influence on failure is very elusive. By comparison, mesoscopic strength is much more sensitive than that at the macroscopic scale. The heterogeneity of mesoscopic strength in an elastic-brittle medium may be represented by a distribution function, $h\left(\sigma_{\mathrm{c}}\right)$. Then damage $D$ can be expressed by the integral

$D=\int_{0}^{\sigma_{\mathrm{s}}} h\left(\sigma_{\mathrm{c}}\right) \cdot \mathrm{d} \sigma_{\mathrm{c}}$

where $\sigma_{\mathrm{c}}$ is normalized mesoscopic strength, $\sigma$ is nominal stress and $\sigma_{\mathrm{s}}=\sigma /(1-D)$ the stress sustained by portion of the material that is still intact. From Eq. (30), the DFD $f=\mathrm{d} D / \mathrm{d} T$ can be derived as

$\frac{\mathrm{d} D}{\mathrm{~d} T}=g(\sigma, D) \cdot \frac{\mathrm{d} \sigma}{\mathrm{d} T}=\frac{h \cdot(1-D)}{(1-D)^{2}-\sigma \cdot h} \cdot \frac{\mathrm{d} \sigma}{\mathrm{d} T}$,

where $h=h(\sigma /(1-D))$. The Weibull distribution $w\left(\sigma_{\mathrm{c}} ; m_{\mathrm{c}}\right)$ is usually adopted as the distribution function $h\left(\sigma_{\mathrm{c}}\right)$. It can be represented by

$w\left(\sigma_{\mathrm{c}}\right)=m_{\mathrm{c}} \sigma_{\mathrm{c}}^{m_{\mathrm{c}}-1} \exp \left[-\left(\sigma_{\mathrm{c}}\right)^{m_{\mathrm{c}}}\right]$

for describing the diversity of mesoscopic strength $[5,18,19]$, where $m_{\mathrm{c}}$ is shape factor or say Weibull modulus. The smaller the Weibull modulus $m_{\mathrm{c}}$ is, the more diverse the strength is. That is to say that the medium is more heterogeneous. Examine damage localization by a criterion similar to that in Eq. (25). The condition of damage localization thus takes the form

$g_{\mathrm{D}} \geqslant \frac{g}{D}$.

This requires

$h^{\prime} \cdot \sigma \cdot D(1-D) \geqslant h \cdot(1-2 D)(1-D)^{2}-h^{2} \cdot \sigma$.

For Weibull distribution, function $g$ is (see Fig. 7)

$g=\frac{m_{\mathrm{c}} \cdot(1-D) \cdot \ln (1-D)}{\sigma \cdot\left[m_{\mathrm{c}} \cdot \ln (1-D)-1\right]}$

and the damage localization criterion becomes

$\left(m_{\mathrm{c}}+1\right) \cdot D_{\mathrm{L}} \geqslant 1+m_{\mathrm{c}} \cdot\left(1-D_{\mathrm{L}}\right) \cdot \ln \left(1-D_{\mathrm{L}}\right)$.

Table 1 gives the critical damage corresponding to different Weibull modula $m_{\mathrm{c}}$, in comparison with assumed critical damage [4]. It is seen that the less the Weibull modulus $m_{\mathrm{c}}$ is (or the more heterogeneous of the mesoscopic strength is) the more difficult is for the materials to under damage localization and failure. This explains the experimental results of failure in rocks [20].

Simulation analyses were carried out by means of a network algorithm [21]. A medium consisting of mesoscopic elements with unique elastic stiffness $K$ was examined. These elements possess but di-
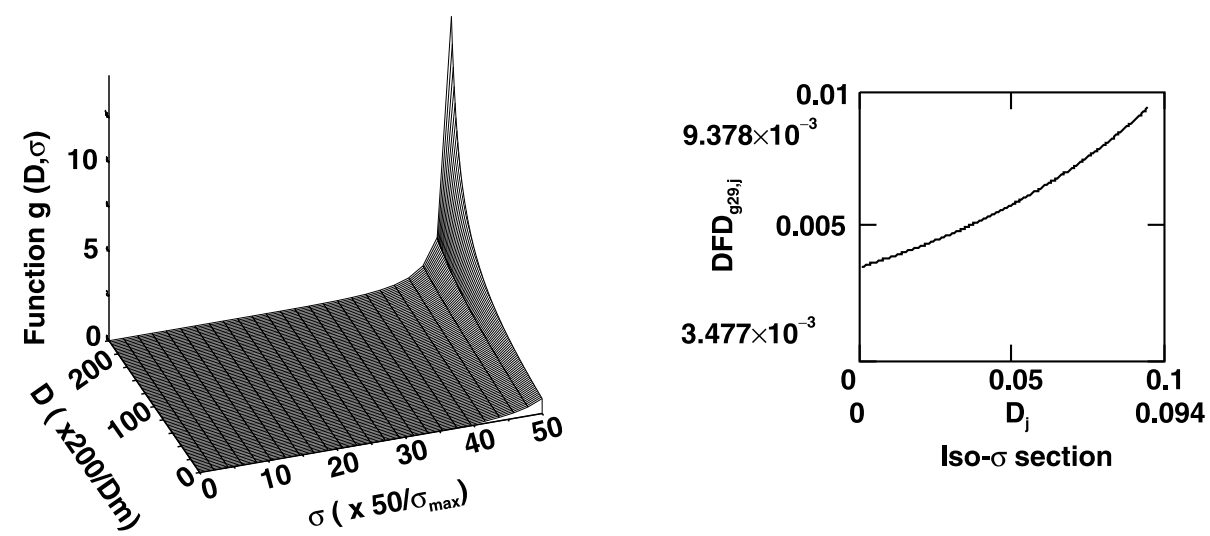

Fig. 7. Function $g(D, \sigma)$ in DFD of heterogeneous brittle media with Weibull distribution and Weibull modulus $m_{\mathrm{c}}=10$. 
Table 1

Critical damage for damage localization $D_{\mathrm{L}}$ in heterogeneous elastic-brittle media with different Weibull modulus $m_{\mathrm{c}}$

\begin{tabular}{lccccc}
\hline Weibull & 15 & 10 & 4 & 2 & 1.6 \\
$\begin{array}{l}\text { modulus } m_{\mathrm{c}} \\
\begin{array}{l}\text { Localization } \\
\text { damage } D_{\mathrm{c}}\end{array}\end{array}$ & .033 & .048 & .11 & .21 & .25 \\
\hline
\end{tabular}

verse strength $\sigma_{\mathrm{c}}$ with Weibull modulus $m_{\mathrm{c}}=5$. The evolution of damage patterns in Fig. 8 shows that the criterion of damage localization does forecast of failure occurrence well.

\subsection{Impact}

The rate-dependent on nature of material spallation under impact has been analyzed by the integral criterion, continuum measure of spallation [17], microstatistical fracture mechanics [6], etc. It was stressed in [22] that quantitative/predictive models are still needed to provide a continuum measure of spalling that might involve nucleationand-growth of microcracks. As mentioned earlier, the dynamic damage function $f$, that bridges mesoscopic nucleation-and-growth of microdamage and continuum damage can be approximated by Eq. (28). A dimensionless version of the function $f$ for spallation is

$f=f(D, \sigma)=G(\sigma) \cdot \gamma \cdot\left(1+\beta \cdot D^{\mu}\right)$,

where $G$ is a dimensionless function of stress. $\gamma$ is a dimensionless parameter denoting the relative nucleation rate, Fig. 9. In this formulation, a characteristic time $t^{*}=c^{*} / C^{*}$ is used to nondimensionalize other parameters. Here, $c^{*}$ and $C^{*}$ are characteristic size and growth rate of microdamage, respectively. More specifically the characteristic time used is $t^{*} \sim 0.527 \mu \mathrm{s}$.

Consider the fitting parameters in Eq. (37) [23]. For a tensile stress $1470 \mathrm{MPa}, G \cdot \gamma \sim(2.7-2.8) \times$ $10^{-3}$ implies that for the characteristic time $t^{*}$ the attained damage is about $10^{-3}$ due to nucleation. The characteristic damage $D^{*}=3.09 \times 10^{-3}$ while the intrinsic Deborah number $\beta$ is as high as $10^{5}-10^{6}$. The corresponding compound rate is

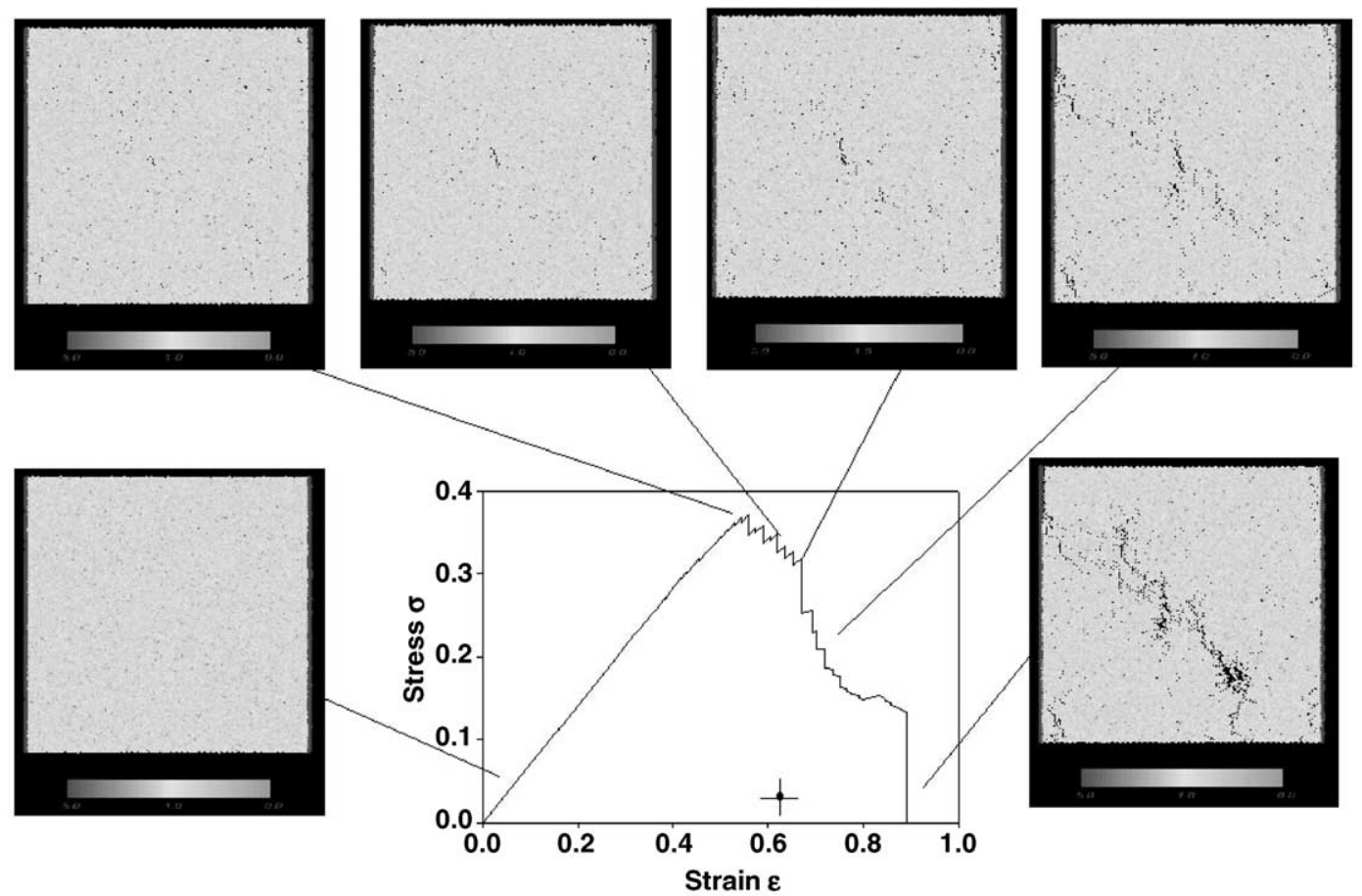

Fig. 8. The simulated stress-strain relation of a heterogeneous model possessing Weibull distribution function with $m_{\mathrm{c}}=5$ and corresponding damage patterns. The cross (+) indicates the damage localization condition (Eq. (23)). 

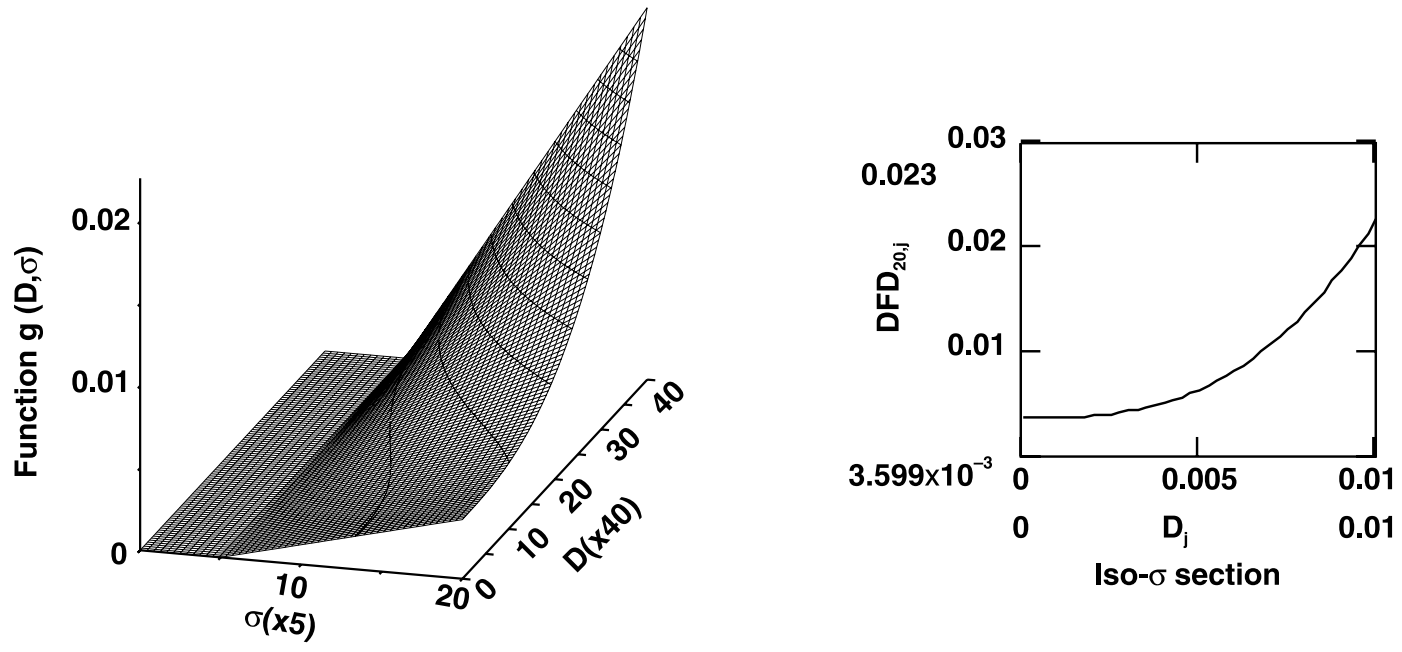

Fig. 9. DFD $f(D, \sigma)$ in the case of impact tests.

Table 2

Critical damage $D_{\mathrm{c}}$, predicted dimensionless life $T_{D_{\mathrm{c}}}$ and $T_{1}(D=1)$ as well as ratio of $T_{D_{\mathrm{c}}} / T_{l}$

\begin{tabular}{llll}
\hline$D_{\max }$ & $D^{*}$ & $2 D^{*}$ & Original data \\
\hline$G \cdot \gamma$ & $2.818 \times 10^{-3}$ & $2.719 \times 10^{-3}$ & \\
$\mu$ & 2.552 & 2.814 & \\
$\beta$ & $2.282 \times 10^{5}$ & $2.246 \times 10^{6}$ & $4.03 \times 10^{-3}$ \\
$D_{\mathrm{c}}$ & $6.49 \times 10^{-3}$ & $4.48 \times 10^{-3}$ & $1.35(0.71 \mu \mathrm{s})$ \\
$T_{D_{\mathrm{c}}}$ & $2.01(1.06 \mu \mathrm{s})$ & $1.46(0.77 \mu \mathrm{s})$ & $2.52(1.33 \mu \mathrm{s})$ \\
$T_{1}$ & $3.68(1.94 \mu \mathrm{s})$ & $2.53(1.33 \mu \mathrm{s})$ & 0.536 \\
$T_{D_{\mathrm{c}}} / T_{1}$ & 0.546 & 0.577 & \\
\hline
\end{tabular}

The figures in brackets are corresponding time.

about $10^{5}-10^{6}$ times the nucleation rate. For further development of damage, compound damage becomes pronounced. Table 2 gives the comparison of the calculated critical damage for damage localization $D_{\mathrm{c}}$, life to damage localization $T_{D_{\mathrm{c}}}$ and life to failure $T_{1}(D=1)$ for fitting ranges $D^{*}$ and $2 D^{*}$. It is worthy noticing that $D_{\mathrm{c}}, D^{*}$ and $G \cdot \gamma$ are in the same order. It implies that as soon as damage due to nucleation develops to a stage characterized by $t^{*}$, to damage localization prevail; this leads to eventual impact failure.

\section{Discussions}

Based on this work, the following points are worthwhile emphasizing.
- Statistical microdamage mechanics is conductive for analyzing material with distributed microdamage.

- The underlying physics of the fundamental evolution equation involve number density associated with microdamage, saturation of microdamage, and moving front of microdamage.

- The DFD bridges the evolution of meso-and macroscopic damage. The condition to de-couple the function and then to reduce the open-endness of these equations at the continuum level is discussed.

- A criterion for damage localization is used by application of intrinsic Deberah number. Eventual failure results when distributed nucleation is overtaken by growth and coalescence of microdamage. 
- Application of statistical microdamage mechanics to heterogeneous medium and spallation under impact are discussed. The results show that the statistical microdamage mechanics and its approximately connected continuum formulations can be used in practice.

- The present approach has also been applied to problems in creep, fatigue, etc. [23,24].

\section{Acknowledgements}

This work is performed under grant supported by the National Natural Science Foundation of China (19891180) and Chinese Academy of Sciences (KJ951-1-201-2).

\section{References}

[1] L.M. Kachanov, Introduction to Continuum Damage Mechanics, Martinus Nijhoff, The Netherlands, 1986.

[2] J. Lemaitre, A. Plumtree, Application of damage concepts to predict creep-fatigue failures, ASME Trans. J. Eng. Mater. \& Tech. 101 (1979) 284-292.

[3] J.L. Chaboche, Continuum damage mechanics, Trans. J. Appl. Mech. 55 (1988) 59-64 (see also pp. 65-72).

[4] J. Lemaitre, A Course on Damage Mechanics, Springer, Berlin, 1992.

[5] D. Krajcinovic, Damage Mechanics, Elsevier, Amsterdam, 1996.

[6] D.R. Curran, L. Seaman, D.A. Shockey, Dynamic failure of solids, Phys. Rep. 147 (1987) 253-388.

[7] S.T. Pantelides, What is materials physics any way?, Phys. Today (September) (1992) 67-69.

[8] D.L. McDowell, Applications of continuum damage mechanics to fatigue and fracture, ASTM STP 1315 (1997) $1-3$.

[9] X.S. Xing, The foundation of nonequilibrium statistical fracture mechanics, Adv. Mech. 21 (1991) 153-168 (in Chinese).
[10] Y.L. Bai, F.J. Ke, M.F. Xia, Formulation of statistical evolution of microcracks in solids, Acta Mech. Sinica 7 (1991) 59-66.

[11] F.J. Ke, Y.L. Bai, M.F. Xia, Evolution of ideal micro-crack system, Sci. China Ser. A 33 (1990) 14471459.

[12] Y.L. Bai, Z. Ling, L.M. Luo, F.J. Ke, Initial development of microdamage under impact loading, ASME Trans. J. Appl. Mech. 59 (1992) 622-627.

[13] W.S. Han, M.F. Xia, L.T. Shen, Y.L. Bai, Statistical formulation and experimental determination of growth rate of micrometer cracks under impact loading, Int. J. Solids Struct. 34 (1997) 2905-2925.

[14] Y.L. Bai, W.S. Han, J. Bai, A statistical evolution equation of microdamage and its application, ASTM STP 1315 (1997) 150-162.

[15] M.F. Xia, W.S. Han, F.J. Ke, Y.L. Bai, Statistical mesoscopic damage mechanics and damage evolution induced catastrophe, Adv. Mech. 25 (1995) 1-40 (in Chinese).

[16] Y.L. Bai, M.F. Xia, F.J. Ke, H.L. Li, Damage field equation and criterion for damage localization, in: R. Wang (Ed.), Rheology of Bodies with Defects, Kluwer Academic Publishers, Dordrecht, 1998, pp. 55-66.

[17] L. Davison, A.L. Stevens, Continuum measures of spall damage, J. Appl. Phys. 43 (1972) 988-994.

[18] W. Weibull, A statistical distribution function of wide applicability, ASME Trans. J. Appl. Mech. 18 (1951) 293297.

[19] B.D. Coleman, On the strength of classical fibersand fiber bundles, J. Mech. Phys. Solids 7 (1958) 60-70.

[20] K. Mogi, Earthquake Prediction, Academic Press, Japan, 1985.

[21] H.Q. Liu, N.G. Liang, M.F. Xia, Modeling and mesoscopic damage constitutive relation of brittle fiber reinforced composites, Sci. China E 43 (1999) 530-540.

[22] M.A. Meyers, Dynamic Behavior of Materials, Wiley, New York, 1994.

[23] Y.L. Bai, J. Bai, H.L. Li, F.J. Ke, M.F. Xia, Damage evolution localization and failure of solids subjected to Impact Loading, Int. J. Impact Eng. 24 (2000) 685-701.

[24] B. Fang, Y.S. Hong, Y.L. Bai, Experimental and theoretical study on numerical density evolution of short fatigue cracks, Acta Mech. Sinica 11 (1995) 144-152. 